\title{
Combining experimental and computational techniques for polymorph screening
}

\author{
D. Šišak Jung ${ }^{a^{*}}$, I. Halasz ${ }^{\mathrm{b}}$, D. McDonagh ${ }^{\mathrm{c},}$ G.M. Day ${ }^{\mathrm{c}}$ \\ ${ }^{a}$ DECTRIS, Switzerland \\ ${ }^{b}$ Division of Physical Chemistry, Ruđer Bošković Institute, Croatia \\ ${ }^{\circ}$ School of Chemistry, University of Southampton, UK \\ *dubravka.sisak@dectris.com
}

\begin{abstract}
Among organic and pharmaceutical molecules, adenine has one of the simplest, small and rigid, molecular structures. However, its crystal structure has been tackled only relatively recently and has yielded two polymorphs, a monoclinic (I) [1] and an orthorhombic (II) [2] form. This fact may come as surprising, given that adenine is a commercial sample, featuring a simple powder X-ray diffraction (PXRD) pattern. However, PXRD patterns of several batches of commercial samples reveal that adenine usually, if not preferably, crystallizes as a mixture of the two polymorphs. Pure phases (I) and (II) can be obtained from a solution or by slurrying, while further polymorph screening revealed no additional crystal forms. Both phases were found to be bench-stable at ambient conditions. However, at temperatures above $280^{\circ} \mathrm{C}$ phase (I) transforms into phase (II).

While this seems to have exhausted the polymorphism of this simple molecule, recent in situ PXRD measurements carried out with an optimized laboratory diffractometer indicate two new phases (III and $\mathbf{X}$ ) at higher temperatures. Phase (III) occurs above $300{ }^{\circ} \mathrm{C}$, and is characterised with a singlepeak PXRD pattern. This phase is stable upon cooling and its structure features randomly oriented adenine layers [3]. At about $200^{\circ} \mathrm{C}$, several low-intensity peaks indicated a new phase, (X). Although these peak positions could be indexed with a triclinic cell of a favourable volume, the data did not allow for cell validation or refinement. To complement the diffraction study, crystal structure prediction was carried out for adenine using quasi-random sampling in a large number of space groups $[4,5]$. This has generated a set of structures energetically close to the two known polymorphs. A few of these computer-generated structures could correspond to the phase (X) observed in the PXRD patterns. Calculations are ongoing.
\end{abstract}

[1] Mahapatra, S.; Nayak, S. K.; Prathapa, S. J.; Guru Row, T. N. Cryst. Growth Des. 2008, 8, 1223.

[2] Stolar T. et al. Cryst. Growth Des. 2016, 16, 3262.

[3] Proffen, T.; Neder, R.B. J. Appl. Cryst. 1997, 30, 171-175.

[4] Case, D. H. et al. J. Chem. Theory Comput. 2016, 12, 910-924.

[5] Price, S. L. et al. Phys. Chem. Chem. Phys. 2010, 12, 8478-8490.

Keywords: crystal structure prediction, in situ, polymorph screening 